\title{
DESIGN OF MATCHED FILTER FOR RADAR APPLICATIONS
}

\author{
Thottempudi Pardhu ${ }^{1}$, A.Kavya Sree ${ }^{2}$ and K.Tanuja ${ }^{3}$ \\ ${ }^{1}$ Assistant.Professor, Department of E.C.E, \\ Marri Laxman Reddy Institute of Technology \& Management, Hyderabad, India \\ 24. pardhu@gmail. com \\ ${ }^{2}$ Department of E.C.E, Marri Laxman Reddy Institute of Technology \& Management, \\ Hyderabad, India \\ alekhyasnehithareddy@gmail.com
}

\begin{abstract}
The aim of this paper is to present the details of signal processing techniques in Military RADARS. These techniques are strongly based on mathematics and specially on stochastic processes. Detecting a target in a noisy environment is a many folds sequential process. The signal processing chain only provides to the overall system boolean indicators stating the presence (or not) of targets inside the coverage area. It is part of the strategical operation of the radar. This paper mainly focuses on Design of Matched filter and generation of chirp Signal.
\end{abstract}

\section{KEYWORDS}

RADAR, Boolean indiactors, Chirp Signal, Matched Filetr, Strategicial Operation

\section{INTRODUCTION}

The use of electromagnetic waves in radar systems imposes some constraints on the overall performances. The basic concept of radars is to detect a target located at a distance denoted $\mathrm{R}$ by sending an electromagnetic wave through an antenna and measuring the time elapsed between the transmitted pulse and its received echo. According to optic/electromagnetic fundamental rules, this time denoted $\mathrm{t}_{0}$ is directly proportional to the light velocity $\mathrm{C}[1]$

$$
\mathrm{t}_{0}=\frac{2 R}{C} \ldots \ldots \ldots \ldots \ldots \ldots
$$

Equation (1) only gives information on the target range. Antenna theory states that the $3 \mathrm{dBbeamwidth}$ of an antenna is related to the carrier wavelength $\lambda$ and the size of the Antenna $\mathrm{D}$ in the given plane

$$
\theta_{3 d B} \cong 70 \frac{\lambda}{D} \ldots \ldots \ldots \ldots \ldots \ldots \ldots \ldots \ldots \ldots \ldots \ldots \ldots \ldots
$$


The direction of the target is directly given by the antenna pointing direction. The accuracy of the direction estimation is increased by augmenting the antenna dimensions.

With a pulsed radar, the signal to be transmitted is composed with a train of individual pulses, each pulse is shifted in time by $T_{R}$ seconds. The pulse repetition frequency $F_{R}$ is defined as

$$
\mathrm{F}_{\mathrm{R}=\frac{1}{\mathrm{TR}}}
$$

This parameter is involved in the calculation of the radar average transmitted power $\mathrm{Pa}$

$$
P_{a}=P_{p} \frac{\tau}{T_{R}}
$$

where $\tau$ is the pulse duration, Ppis the peak power. The duty cycle, defined as $\frac{\tau}{T_{R}}$, provides information on radar average operation, one thousand is a common value.

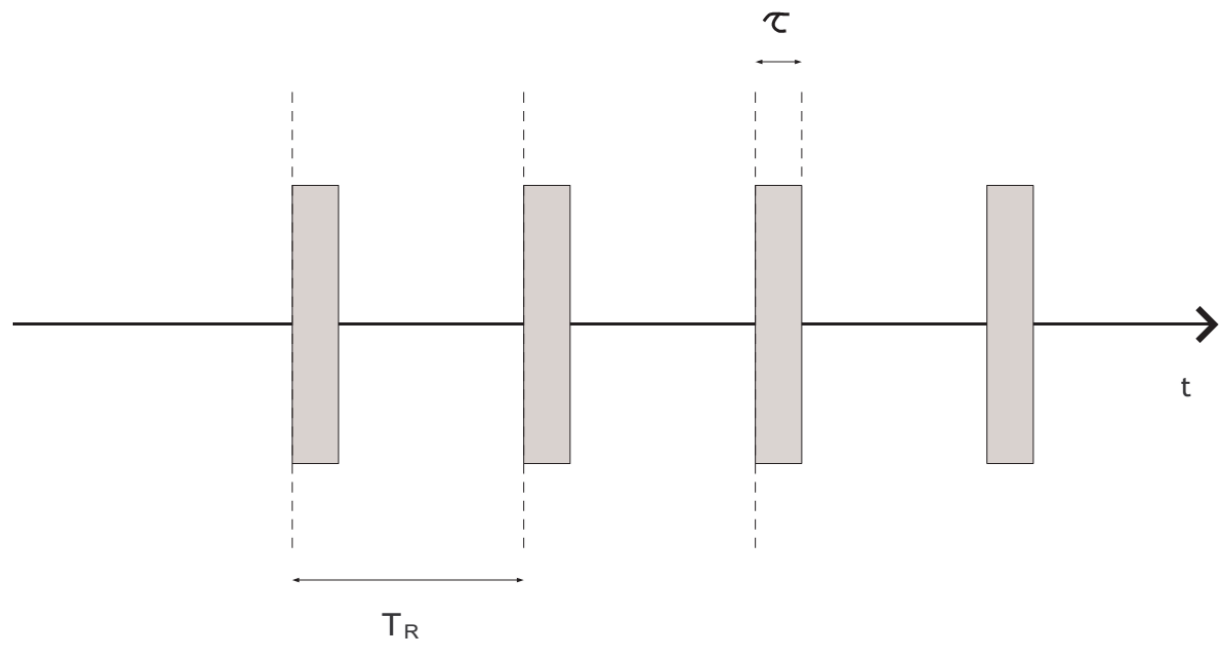

fig 1: Pulses Brust diagram

For a pulsed radar, a new notion that does not exist in continuous-wave radar is defined: the range-discrimination factor. It is used in situations when two distinct targets are located in the same direction but at different ranges $\left(R_{1} \neq R_{2}\right)$. This means that both targets are located inside the antenna main-lobe. Thus, if the two targets respond to the transmitted signal by producing an echo, these echos are shifted in time such that

$$
\Delta t=\frac{2 R_{1}}{c}-\frac{2 R_{2}}{c}=\frac{2}{c} \Delta R
$$

Each pulse has a duration equal to $\tau$ and if $\Delta \mathrm{t}<\tau$, then the two echos overlap in time.If we only consider signal amplitude, the two signals may cancel each other in some points in time. One 
target may be hidden in such situation, that is not acceptable in a military context. The rangediscrimination factor is defined as

$$
\Delta R=\frac{c \tau}{2}
$$

Using the same concept, an angular-discrimation factor is defined. This radar parameter applies to both vertical (site) and horizontal (azimuthal) directions. It is expressed as

$$
\Delta \theta=1.2 \frac{\lambda}{D}
$$

Section 1 gives introduction about the signal processing section 2, 3 deals with pulse compression and principle of pulse compression, section 4 gives information about generation of chirp signal, section 5 briefs us about Matched filter, section 6 gives results and analysis of the problem and section 7 concludes and gives future scope.

\section{PULSE COMPRESSION}

The pulse compression block is the first stage of the signal processing chain. At the input of the module, the received signal is very noisy and almost looks like white noise .The pulse compression technique tries to make the transmitted signal visible and dominant over the background noise. Unlike the pulses depicted in figure .1, the transmitted signal does not consist of basic rectangular-like pulses with constant amplitude and duration $\tau$. The main reason is due to electromagnetic laws that do not permit low-frequencies signal to be sent over the air. Therefore there is a need to shift the signal on a carrier signal. Moreover, the system generates internal noise. The noise power decays in 1/f. Thus, system self-generated noise is inversely proportional to the frequency. This gives a second good reason to use a carrier signal to transmit the signal. A basic quadratic demodulator recovers the signal from the RF modulation. The detected signal is shifted back to the baseband domain. Using a specific receiving filter, the output produces a triangular-like signal which maximum value leads to the target range.[2][3]

\section{PRINCIPLE OF COMPRESSION}

Pulse Compression is involved in radar systems where both maximum detection range and range discriminator factor are of importance. We know that maximum detection range $\mathrm{R}_{\max }$ is proportional to the pulse duration $\tau$ and the range discriminator factor $\mathrm{d}$ is $d=\frac{c \tau}{2}$ and is must be minimized to ensure multiple targets detection. One first idea could be to increase the peak power while keeping the pulse duration to a low value, thus increasing the maximum range detection. However, due to hardware constraints, this solution is not feasable. The most popular solution consists in the use of particular pulses, called compressed pulses.

Compressed pulses are pulses that have the particularity to have a non-zero duration $\mathrm{T}$ at Transmitting point such that $\mathrm{T}>>\tau$. At the receiving point, their duration is set back to $\tau$ after filtering. We define

$$
\rho=T / \tau
$$


as the compression rate. Basic rectangular pulses on a carrier do not allow such results. It is necessary to add a modulation or coding scheme on the signal phase. The most popular coding scheme is the so-called chirp scheme.

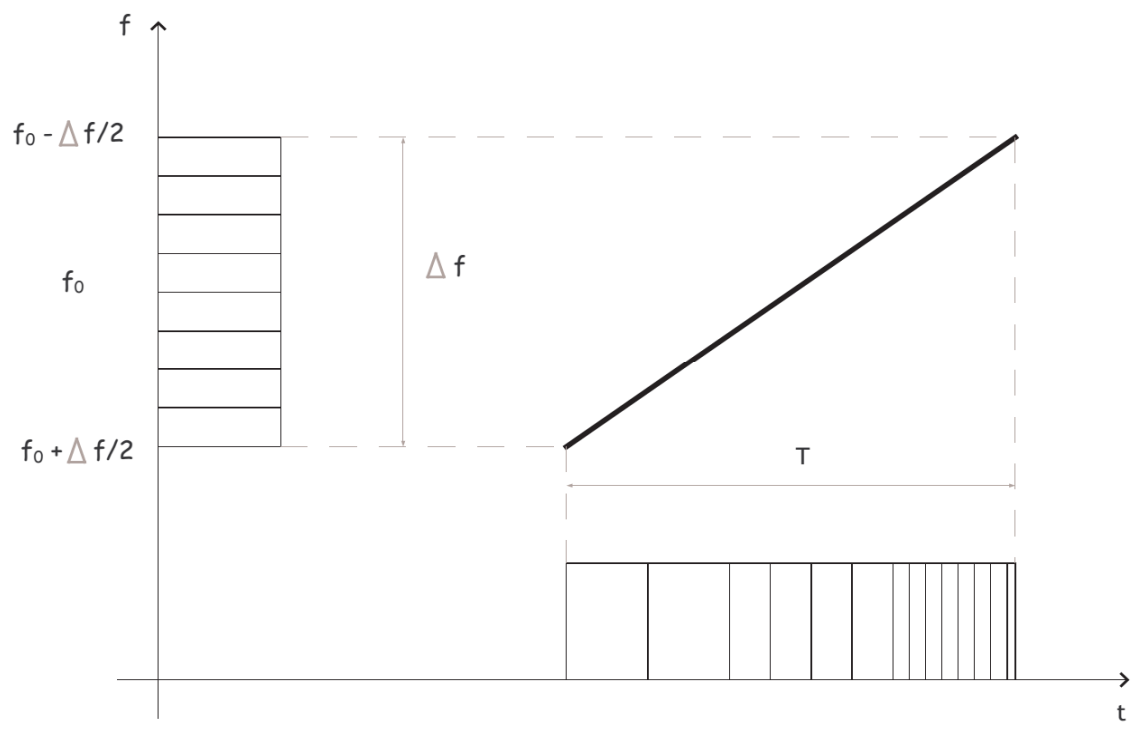

Figure.2 : Pulse Compression principle

Figure. 2 depicts the basic principle of the pulse compression. The frequency of a signal (a pure tone) is linearly modulated in the frequency range $[\mathrm{f} 0-\Delta \mathrm{f} / 2, \mathrm{f} 0+\Delta \mathrm{f} / 2]$ over the pulse duration $\mathrm{T}$ where $\mathrm{f}_{0}$ is the carrier frequency. Thus, the transmitted signal is a repetition of frequency modulated pulses of duration T.[2][3]

\section{GENERATION OF SignAL}

Several techniques exist when it comes to generate the transmitted signal. Signal generation can be passive or active. It can also be digital or analog. Figure 3 describes the passive method of producing a compressed signal. This method is defined as passive because a specific filter is used to produce the frequency modulation from a basic rectangular pulse. The MRR radar uses digital computation. The use of digital calculation allows to store several pulses in a memory bank and to select a given pulse at any given instant according to the situation. Indeed, compressed pulses have specific characteristics that have a direct impact on the overall radar performance. For instance, pulse duration $\mathrm{T}$ and $\tau$ impacts the covered range or the range discriminator; the frequency drift $\Delta \mathrm{f}$ has an impact on the Doppler filtering and can also be selected.[5] 
Electrical and Electronics Engineering: An International Journal (ELELIJ) Vol 3, No 4, November 2014

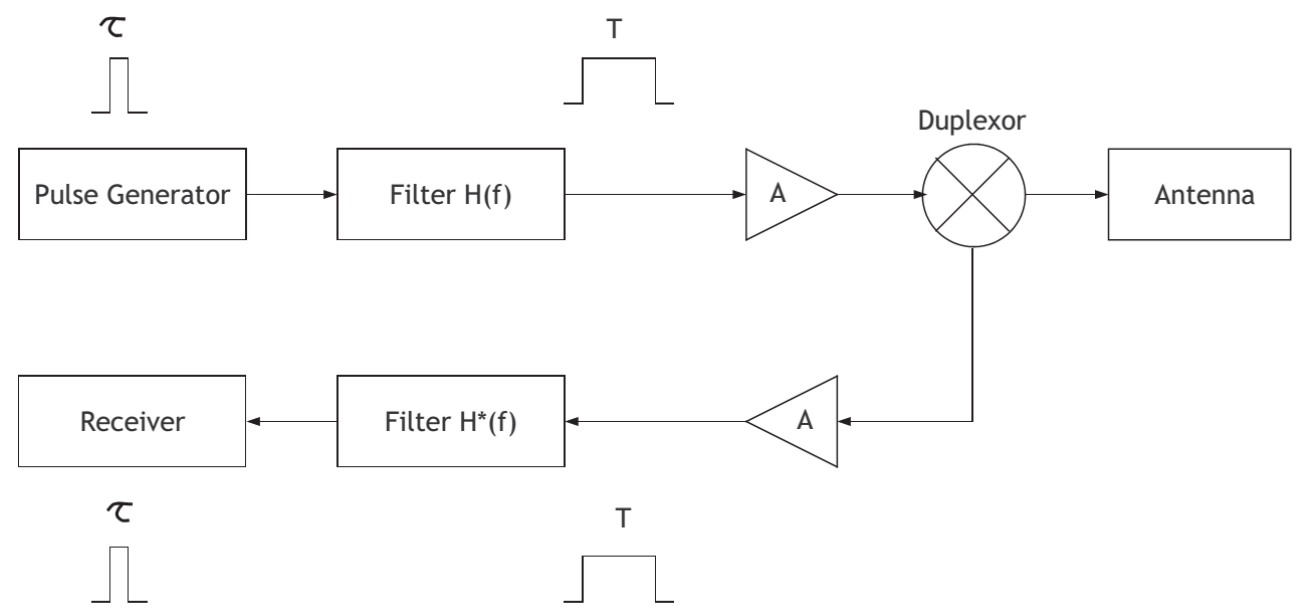

Figure.3 Pulse Compression Signal generation

\section{Chirp Signal: Mathematical Approach}

The chirp signal is a signal where the instantaneous frequency linearly varies within the duration $\mathrm{T}$ of the transmitted pulse. We define the phase variation rate in $[\mathrm{rad} / \mathrm{s}]$ as $\mu=2 \pi \Delta \mathrm{f} / \mathrm{T}$ and we build the signal $\mathrm{x}(\mathrm{t})$ as follow

$$
x(t)=\cos \left(\omega_{0} t+\frac{1}{2} \mu t^{2}\right),-\frac{T}{2} \leq t \leq \frac{T}{2}
$$

Where $\omega_{0}$ is the carrier frequency of the transmitted signal. Its spectrum is computed using the Fourier Transform as [6]

$$
\begin{aligned}
X(f) & =F T\{x(t)\} \\
& =\int_{-\frac{T}{2}}^{\frac{T}{2}} \cos \left(\omega_{0} t+\frac{1}{2} \mu t^{2}\right) \exp (-j 2 \pi f t) d t .
\end{aligned}
$$

After calculations we obtain

$$
X(f)=\frac{1}{2} \sqrt{\frac{\pi}{\mu}} \exp \left(-\frac{j\left(\omega_{0}-\omega\right)^{2}}{2 \mu}\right)\left[C\left(x_{1}\right)+j S\left(x_{1}\right)+C\left(x_{2}\right)+j S\left(x_{2}\right)\right],
$$

Where 
Electrical and Electronics Engineering: An International Journal (ELELIJ) Vol 3, No 4, November 2014

$$
\begin{gathered}
x_{1}=\frac{\frac{\mu T}{2}+\left(\omega_{0}-\omega\right)}{\sqrt{\pi \mu}}, \\
x_{2}=\frac{\frac{\mu T}{2}-\left(\omega_{0}-\omega\right)}{\sqrt{\pi \mu}}, \\
C(x)=\int_{0}^{x} \cos \left(\frac{\pi}{2} y^{2}\right) d y, \\
S(x)=\int_{0}^{x} \sin \left(\frac{\pi}{2} y^{2}\right) d y .
\end{gathered}
$$

Integrals $\mathrm{C}(\mathrm{x})$ and $\mathrm{S}(\mathrm{x})$ are called Fresnel integrals. Using the fact that the signal is composed of successive pulses transmitted every $\mathrm{TR}=1 / \mathrm{FR}$, the signal spectrum is a discontinuous spectrum composed of regularly spaced narrow rays (every $F_{R}$ in the frequency domain). The spectrum envelope module is given by

$$
|X(f)|=\frac{1}{2} \sqrt{\frac{\pi}{\mu}}\left[\left(C\left(x_{1}\right)+C\left(x_{2}\right)\right)^{2}+\left(S\left(x_{1}\right)+S\left(x_{2}\right)\right)^{2}\right]
$$

It is possible to show that for $\rho=\mathrm{T} / \tau=\mathrm{T} \times \Delta \mathrm{f}$, the envelope module is approximately constant within the considered bandwidth $\Delta \mathrm{f}$

$$
|X(f)|_{T \times \Delta f>>1} \cong \sqrt{\frac{\pi}{\mu}}, f_{0}-\frac{\Delta f}{2} \leq t \leq f 0+\frac{\Delta f}{2} .
$$

In a realistic scenario, it is necessary to consider a complex signal as the noise is modeled as complex. It affects both amplitude and phase.

$$
x(t)=\exp \left(j\left(\omega_{0} t+\frac{1}{2} \mu t^{2}\right)\right),-\frac{T}{2} \leq t \leq \frac{T}{2} .
$$

Hence, the transmitted pulse train signal, composed of Np pulses, is written as[8]

$$
x_{T X}=\sum_{i=0}^{N_{p}} x(t-i T) .
$$




\section{MATCHED FILTER}

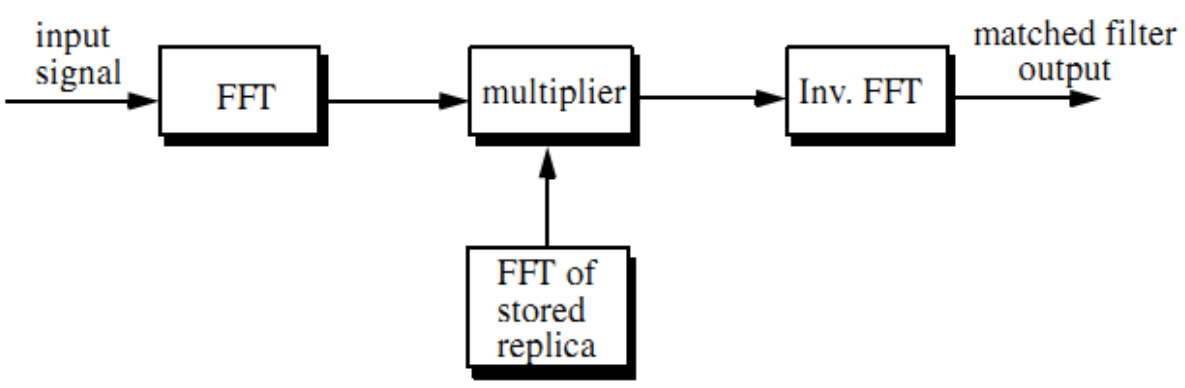

Figure.4 : block diagram of matched Filter

The above diagram represents the block diagram of Matched Filter.

As any signal transmitted over the air, the chirp signal encounters noise in its two way trip from the transmitted antenna to the target and back to the antenna. In order to maximize the signal-tonoise ratio at the receiving stage, the matched filter is the optimal solution Here we give a summarized overview. For a received signal $y(t)$ which is a time-shifted (delayed) replica of the transmitted signal with additive noise $\mathrm{n}(\mathrm{t})$

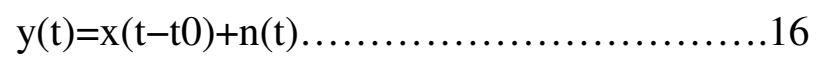

The frequency response function of the linear, time-in variant filter which maximize the output peak-signal-to-mean-noise (power) ratio for a given input signal-to-noise ratio is given by:

$$
H(f)=G_{a} Y^{*}(f) \exp \left(-j 2 \pi \mathrm{ft}_{0}\right) \ldots \ldots \ldots \ldots \ldots \ldots 17
$$

where:

- $\quad Y(f)$ is the Fourier transform of $y(t): Y(f)=\int_{-\infty}^{+\infty} y(t) \exp (-j 2 \pi f t) d t$

- $\quad$ t0: fixed value of time at which the signal is oberved to be maximum (equals the round trip delay of the transmitted signal)

- Ga: filter gain (generally set to unity)

The noise that accompanies the signal is assumed to be stationary and to have a uniform spectrum (white noise). It does not need to be Gaussian. In the time domain, using inverse Fourier transform, the matched filter impulse response can be expressed as follow using the fact that $Y *(f)=Y(-f)$ :

$$
\begin{aligned}
\mathrm{h}(\mathrm{t}) & =\int_{-\infty}^{+\infty} H(f) \operatorname{expj} 2 \pi f t d f \\
& =\mathrm{G}_{\mathrm{a}} \int_{-\infty}^{+\infty} Y *(f) \exp [-j 2 \pi f(t 0-t)] d f \\
& =\mathrm{G}_{\mathrm{a}} \int_{-\infty}^{+\infty} Y(f) \exp [j 2 \pi f(t 0-t)] d f
\end{aligned}
$$




$$
=\mathrm{Ga} \cdot \mathrm{y}(\mathrm{t} 0-\mathrm{t})
$$

The impulse response of the matched filter is simply the image of the received signal; that is, it is the same as the received signal but run backward in time starting from instant $t_{0}$.However, since the noise $\mathrm{n}(\mathrm{t})$ is an unknown signal, the filter is matched to the transmitted signal $\mathrm{x}(\mathrm{t})$ :

$$
h(t)=G a \cdot x\left(t_{0}-t\right)
$$

The output of the matched-filter is expressed as the convolution in the time domain of the received signal with the matched-filter impulse response:

$$
\begin{aligned}
& =\int_{-\infty}^{+\infty} y(\tau) \cdot h(t-\tau) d \tau \ldots \ldots \ldots \ldots \ldots \ldots \ldots \ldots \ldots \ldots \ldots . \ldots . \ldots \ldots \\
& \quad=\int_{-\infty}^{+\infty}(x(\tau)+n(\tau)) \cdot x(t 0-t-\tau) d \tau \\
& =\int_{-\infty}^{+\infty} x(\tau) \cdot x(t 0-t-\tau) d \tau+\int_{-\infty}^{+\infty} n(\tau) \cdot x(t 0-t-\tau) d \tau \ldots \ldots 21 \\
& =\mathrm{z}(\mathrm{t})+\mathrm{w}(\mathrm{t})
\end{aligned}
$$

Where $z(t)$ represents the noise-free output from the matched filter and $w(t)$ represents the filtered noise. Note that since the transmitted signal spans a wide frequency range, the matched filter cuts out some signal as well as some background noise since it has a limited bandwidth.[7]

In radar signal theory, the matched filter output is affected by the Doppler Effect. The apriori unknown frequency shift introduced by the Doppler effect can also be seen in the time domain as an additional def, say tD. Therefore, since both target range and target Doppler shift are unknown to the system, a bank of matched filters is used to determine the overall delay. Doppler filtering output, i.e. a frequency shift, helps to best estimate the target range by subtracting the delay $t_{D}$ to the total delay.

\section{RESULTS AND ANALYSIS}

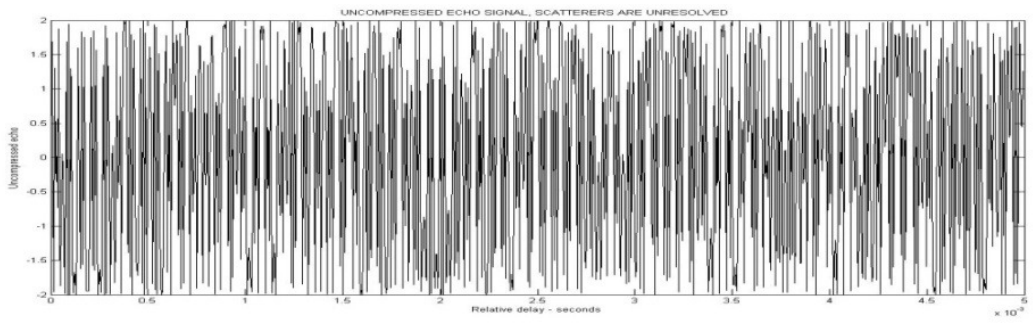

Figure.5 : Generated Chirp Signal

The above diagram represents the generated signal which is uncompressed echo received by the RADAR from $150 \mathrm{~km}$ Distance. 
Electrical and Electronics Engineering: An International Journal (ELELIJ) Vol 3, No 4, November 2014

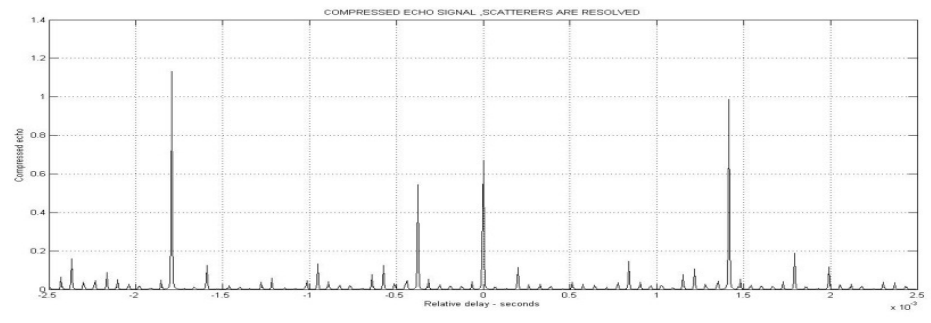

Figure.6: Compressed Echo

The above diagram represents the compressed echo which is passed through Kizer Filter.
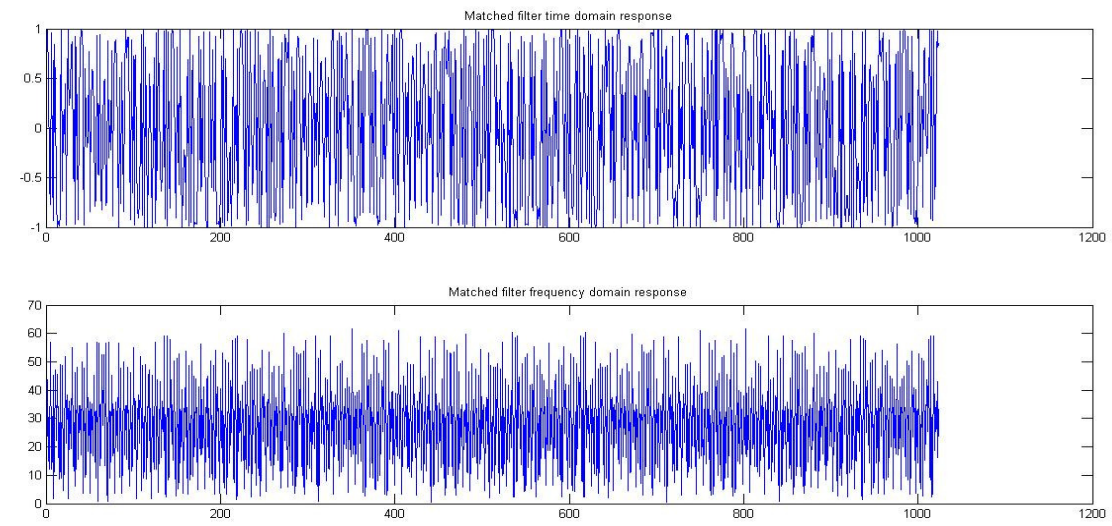

Figure.7: Matched filter time and frequency domain responses

The above Figure Represents the output of Matched filter.

\section{CONCLUSION AND FUTURE SCOPE}

This paper aims to present in details the signal processing techniques employed in a military radar. These techniques are strongly based on mathematics and specially on stochastic processes. Detecting a target in a noisy environment is a many folds sequential process. The signal processing chain only provides to the overall system boolean indicators stating the presence (or not) of targets inside the coverage area. It is part of the strategical operation of the radar.

The selection of signal procesing techniques according to the radar performance requirements is one of the most important step in military radar design. Signal processing, as we have seen throughout this paper, provides figures that tactical leaders take into account when looking for performances and capabilities. Nowadays, digital processing allows fast and efficient computation. The basic example is the use of FFT and IFFT to perform time-convolution (filtering).

As a future work, one could focus on new antenna techniques that allow multiple beam to operate simultaneously. These new antennas provides supplementary ressources to track multiple targets. They are based on flat electronically steered arrays (SMBAA). We can use this process in generating Various Clutters. 
Electrical and Electronics Engineering: An International Journal (ELELIJ) Vol 3, No 4, November 2014

\section{REFERENCES}

[1] F. Athley. Space-Time Parameter Estimation in Radar Array Processing.PhD thesis, Chalmers University of Technology, Department of Signals and Systems, G*oteborg, Sweden, 2003.

[2] David K. Barton. Radars volume. In Pulse Compression,volume3.Artech House, Inc., 1975.

[3] David K. Barton. Radars volume. In Pulse Compression,volume7.Artech House, Inc., 1975.

[4] Henry W. Cole. Understanding Radar. COLLINS, 1985

[5] North D.O. An analysis of the factors which determine signal/noise discrimination in pulsed-carrier systems.Reprinted in Proc. IEEE, 51:1016-1027, July 1963.

[6] Stephen S. Johnston. Target fluctuation models for radar system design and performance analysis : An overview of three papers. IEEE Transactions onAerospace and Electronic Systems, 33(2), April 1997.

[7] Merrill I. Skolnik. Introduction to Radar Systems. McGraw-Hill Book Company, 2nd edition, 1980.

[8] S. Watts. Cfar detection in spatially correlated sea clutter. EUREL meeting on Radar and Sonar Signal Processing, Peebles, July 1998.

\section{AUTHORS PROFILE}

T.Pardhu was born in Luxettipet village in Adilabad District. He completed B.Tech in MLR Institute of Technology in the stream of Electronics and Communications Engineering in 2011. He has done his Master's degree (M.Tech) in Embedded Systems from Vignan University, Vadlamudi in 2013.He has done Project in Research Centre IMARAT, Hyderabad as Project Intern. He is Working as Assistant Professor in Marri Laxman Reddy Institute of Technology \& Management.His interested fields are Digital signal processing, RADAR communications, Embedded systems, implementation of signal processing on applicationsin FPGA.

N.Alekhya Reddy was born in Nellore. She is studying her Bachelor's Degree (B.Tech) in MLR Institute of Technology in the stream of Electronics and Communication Engineering. Her Research interest Fields includes Signal Processing, Low power VLSI.
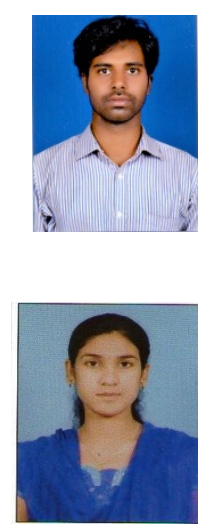